\title{
Diapycnal Transport near a Sloping Bottom Boundary
}

\author{
R. M. Holmes ${ }^{\mathrm{a}, \mathrm{b}, \mathrm{c}}$ AND TREVOR J. MCDOUGALL ${ }^{\mathrm{a}}$ \\ ${ }^{\mathrm{a}}$ School of Mathematics and Statistics, University of New South Wales, Sydney, New South Wales, Australia; ${ }^{\mathrm{b}}$ Climate Change \\ Research Centre, University of New South Wales, Sydney, New South Wales, Australia; ${ }^{c}$ Australian Research Council Centre of \\ Excellence for Climate Extremes, University of New South Wales, Sydney, New South Wales, Australia
}

(Manuscript received 25 March 2020, in final form 24 August 2020)

\begin{abstract}
The diapycnal motion in the stratified ocean near a sloping bottom boundary is studied using analytical solutions from one-dimensional boundary layer theory. Bottom-intensification of the diapycnal mixing intensity ensures that in the stratified mixing layer (SML), where isopycnals are relatively flat, the diapycnal motion is downward toward denser fluid. In contrast, convergence of the diffusive buoyancy flux near the seafloor drives diapycnal upwelling in what we define as the bottom boundary layer (BBL). Much of the one-dimensional BBL is characterized by a stratification only slightly reduced from that in the SML because the maximum in the buoyancy flux at the top of the BBL, where the diapycnal velocity changes sign, must occur in well-stratified fluid. The diapycnal upwelling in the BBL is determined by variations not only in the magnitude of the buoyancy gradient but also in the curvature of isopycnals. The net diapycnal upwelling is concentrated in the bottom half of the BBL where the magnitude of the buoyancy gradient changes most rapidly. The curvature effect drives upwelling near the seafloor that only makes a significant contribution to the net upwelling for steep slopes. The structure of the diapycnal velocity in this stratified BBL differs from the case of a turbulent well-mixed BBL that has been assumed in some recent theoretical studies on bottom-intensified mixing. This work therefore extends recent theories in a way that should be more applicable to abyssal ocean observations where well-mixed BBLs are not common.
\end{abstract}

KEYWORDS: Abyssal circulation; Diapycnal mixing; Lagrangian circulation/transport; Mass fluxes/transport; Boundary layer

\section{Introduction}

The turbulent bottom boundary layer in the abyssal ocean is thought to play a key role in the conversion of dense bottom waters into lighter water (de Lavergne et al. 2016; Ferrari et al. 2016; McDougall and Ferrari 2017, hereafter MF17; de Lavergne et al. 2017). The observed bottom-intensification of internal-wavedriven mixing above rough topography in the abyssal ocean (Toole et al. 1994; Polzin et al. 1997; Waterhouse et al. 2014) drives the densification, or diapycnal downwelling, of waters within so-called "abyssal mixing layers" extending hundreds of meters above the topography. In contrast, due to the insulating bottom boundary condition the buoyancy flux must converge within a thinner layer right next to the seafloor, driving a lightening of waters and thus diapycnal upwelling. This motivates a definition of a bottom boundary layer (BBL), used throughout this article, as that layer over which the diapycnal transport is upward. Globally, the diapycnal upwelling within the BBL must exceed the diapycnal downwelling in the stratified mixing layer (SML) above the BBL in order to drive a net diapycnal upwelling that balances the formation of dense water at the high latitudes. For example, Thurnherr et al. (2020) suggest that the majority of the upwelling of Antarctic bottom water in fracture zone canyons in the Brazil Basin occurs within thin BBLs along the canyon walls. However, despite the inferred importance of this BBL diapycnal upwelling it has yet to be observed directly.

Several recent theoretical studies on the role of the BBL in the global diapycnal circulation (Holmes et al. 2018; MF17)

Corresponding author: R. M. Holmes, ryan.holmes@unsw. edu.au have modeled the BBL as an idealized fully mixed layer where the buoyancy flux increases linearly with distance away from the seafloor. This fully mixed BBL was assumed to host all of the global diapycnal upwelling, significantly more than the net upwelling of bottom waters by an "amplification factor" that may reach factors of 3-5. However, such fully mixed BBLs are rarely observed in the ocean (e.g., van Haren 2017; Thurnherr et al. 2020), raising the question of whether the model of MF17 is appropriate for studying abyssal water-mass transformation.

Useful theoretical solutions in which some of these issues can be explored are available from steady-state one-dimensional boundary layer theory (e.g., Wunsch 1970; Phillips et al. 1986; Thorpe 1987; Garrett 1990, 1991; Garrett et al. 1993; Callies 2018). While this theory does not fully capture much of the complexity in near-bottom flows, including processes such as symmetric instability, baroclinic instability, tidal variability, and topographic variability (e.g., Allen and Newberger 1998; Umlauf and Burchard 2011; Kunze et al. 2012; Umlauf et al. 2015; Wenegrat et al. 2018; Callies 2018; Ruan et al. 2019; Ruan and Callies 2020), it provides a useful starting point to discuss diapycnal fluxes that is more dynamically consistent than the assumption of a fully mixed layer. The connection between diapycnal transport and boundary layer theory has been discussed by Garrett (2001). He introduced a formula for the diapycnal velocity that depends not only on variations in the magnitude of the buoyancy gradient and diapycnal diffusivity, but also on the geometry, or curvature, of isopycnals. However, he did not further explore the partitioning between these contributions.

The intention of this article is to clarify the connections between BBL turbulence and the diapycnal transport in the 
abyssal ocean by 1) providing a clear and simple explanation as to why diapycnal upwelling is expected near the boundary, 2) examining several driving forces of diapycnal transport in the one-dimensional BBL, and 3) comparing and contrasting the MF17 fully mixed model of the BBL with the BBL structure obtained from one-dimensional boundary layer theory. Our hope is to better connect the recent theoretical results to observations. We will also discuss some generalizations of these results to cases where upslope variations in the turbulent buoyancy flux or isopycnal structure are permitted.

\section{Diapycnal transport and BBL upwelling}

\section{a. The diapycnal velocity}

We begin with the Boussinesq buoyancy equation,

$$
\frac{D b}{D t}=-\nabla \cdot \mathcal{B}
$$

where $b$ is buoyancy and $\mathcal{B}$ is a turbulent, nonadvective, buoyancy flux per unit area. Throughout most of this article we will consider a diffusive flux,

$$
\mathcal{B}=-\kappa \nabla b,
$$

where $\kappa$ is a turbulent eddy diffusivity. However, it should be noted that within a fully mixed bottom layer (as used in MF17) the turbulent buoyancy flux is not related to the local buoyancy gradient.

The diapycnal velocity $\omega$ (or fluid volume flux across an isopycnal toward lighter densities per unit area of the isopycnal) can be written (Marshall et al. 1999)

$$
\omega=-\frac{1}{|\nabla b|} \nabla \cdot \mathcal{B} .
$$

Diapycnal upwelling occurs when the turbulent buoyancy flux per unit area is convergent resulting in buoyancy gain. The factor of $|\nabla b|^{-1}$ is present because fluid parcels move more rapidly across widely spaced isopycnals given a similar rate of buoyancy gain or loss. A finite value of $|\nabla b|$ is needed to define the diapycnal velocity as otherwise the location of the given isopycnal across which $\omega$ transports volume is ambiguous.

Equation (3) suggests that one would expect diapycnal upwelling to occur within some layer immediately adjacent to the seafloor. To see this, consider a nonzero turbulent diffusivity $\kappa$ and a stable stratification some distance $H$ away from the boundary. The buoyancy flux $\mathcal{B}$ will have a component that is directed toward the boundary at this distance. The zero normal buoyancy flux condition at the seafloor (or an inward pointing geothermal buoyancy flux) then implies a convergence of the turbulent buoyancy flux and upwelling, $\omega>0$. This intuition will be put on a firmer footing in section 2c. However, we first present several simple decompositions of Eq. (3).

\section{b. A geometric decomposition of the diapycnal velocity}

By writing the diffusive buoyancy flux $\mathcal{B}=\mathcal{B} \hat{\mathbf{n}}$ in terms of its magnitude $\mathcal{B}$ and the isopycnal normal $\hat{\mathbf{n}}$ and using the chain rule the diapycnal velocity in Eq. (3) can be decomposed into two terms,

$$
\omega=\underbrace{\frac{1}{|\nabla b|} \hat{\mathbf{n}} \cdot \nabla \mathcal{B}}_{\omega_{\mathcal{B}}}+\underbrace{\frac{\mathcal{B}}{|\nabla b|} \nabla \cdot \hat{\mathbf{n}}}_{\omega_{g}} .
$$

Equation (4) shows that spatial variations in the buoyancy flux magnitude, associated with $\omega_{\mathcal{B}}$, are not the only contributor to diapycnal transport. The additional factor $\omega_{g}$, which for a diffusive flux where $\mathcal{B}=\kappa|\nabla b|$ can also be written

$$
\omega_{g}=\kappa \nabla \cdot \hat{\mathbf{n}},
$$

arises from isopycnal curvature. The factor $\omega_{g}$ is zero when isopycnals are flat even if the stratification varies. This decomposition has been discussed by Garrett [2001, his Eq. (11)].

The diapycnal velocity can also be decomposed into components associated with variations in the diffusivity and the buoyancy gradient, respectively [substitute Eq. (2) in Eq. (3) and use the chain rule],

$$
\omega=\underbrace{\hat{\mathbf{n}} \cdot \nabla \kappa}_{\omega_{\kappa}}+\underbrace{\frac{\kappa}{|\nabla b|} \nabla^{2} b}_{{ }^{\omega} \nabla^{2} b} .
$$

Equations (6) and (4) are related through $\omega_{\mathcal{B}}=\omega_{|\nabla b|}+\omega_{\kappa}$, where

$$
\omega_{|\nabla b|}=\frac{\kappa}{|\nabla b|} \hat{\mathbf{n}} \cdot \nabla(|\nabla b|),
$$

is associated with gradients in isopycnal layer thickness. The decomposition in Eq. (6) will prove useful as the first term is largely responsible for downwelling in the SML (where $\kappa$ decreases with height) while the second term is largely responsible for upwelling in the BBL (where the slope-normal buoyancy gradient must go to zero at the boundary). These decompositions will be discussed in more detail in section 3 using solutions from one-dimensional boundary layer theory.

\section{c. Net diapycnal transport across an isopycnal near the boundary}

Returning to the total diapycnal velocity [Eq. (3)], in this section we revisit the MF17 derivation of the net diapycnal transport across an isopycnal near the boundary in order to establish under what conditions net upwelling should be expected. Here we will generalize the MF17 result by considering an arbitrarily curved isopycnal geometry (accounting for any diapycnal transport associated with $\omega_{g}$ and lifting the assumption of a fully mixed BBL). We also include the diapycnal transport term associated with convergences or divergences of the buoyancy flux across isopycnals inside the BBL that was neglected by MF17. For convenience, we will consider a coordinate system aligned with a sloping abyssal boundary (with slope to the horizontal of $\tan \theta$ ) where $z$ is the slope-normal coordinate (with origin at the boundary), $y$ is the upslope direction and $x$ is the along-slope direction (see Fig. 1). The net volume transport across an isopycnal with buoyancy value $b$ within a distance $h$ of the boundary, $\mathcal{E}(b, h, t)$, can be derived by considering the region between that isopycnal and the 


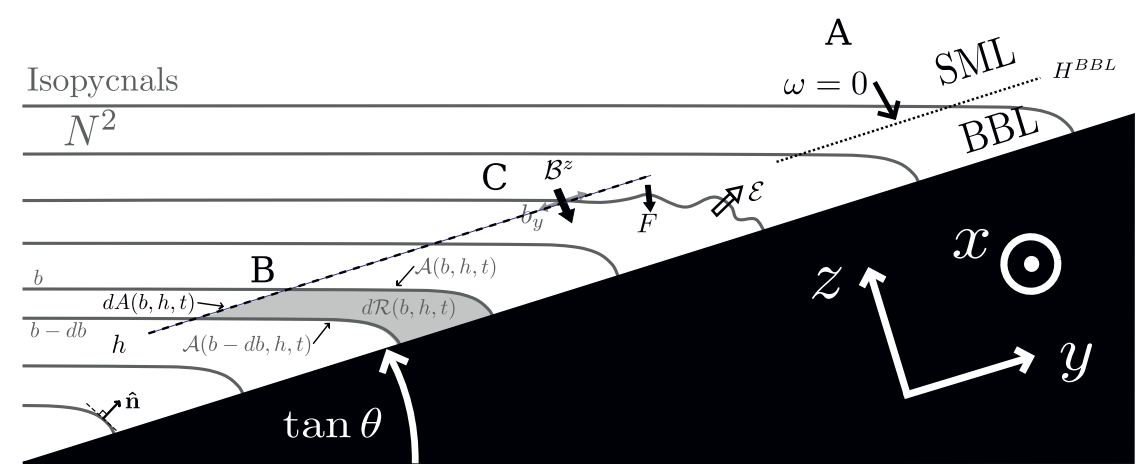

FIG. 1. A schematic illustrating the rotated coordinate system used in this article, where $z$ is the slope-normal direction (where the slope is $\tan \theta$ ), $y$ is the upslope direction, and $x$ is the along-slope direction. In the one-dimensional solutions the far-field interior stratification is $N^{2}$. The top of the BBL is defined by the height $z=H^{\mathrm{BBL}}$ at which the diapycnal velocity $\omega$ is zero (black dotted line in A). The gray shaded region denotes an infinitesimal region between two isopycnals and within a distance $h$ of the boundary (black dashed line in B) over which the net diapycnal transport is calculated (also see appendix A). This yields a relationship [Eq. (14)] between the diapycnal transport across the isopycnal $\mathcal{E}$, the diffusive buoyancy flux across that isopycnal $F$, and the diffusive buoyancy flux toward the boundary at the height $h$, $\mathcal{B}^{z}$, that is valid for an arbitrary isopycnal geometry $(\mathrm{C})$.

isopycnal with buoyancy value $b-d b[d \mathcal{R}(b, h, t)$, gray region in Fig. 1], where $d b$ is an infinitesimal buoyancy difference. Integrating Eq. (3) over the isopycnal out to a slope-normal distance $z=h$ away from the boundary,

$$
\mathcal{E}(b, h, t)=\iint_{\mathcal{A}(b, h, t)} \omega d \mathcal{S}=-\iint_{\mathcal{A}(b, h, t)} \frac{\nabla \cdot \mathcal{B}}{|\nabla b|} d \mathcal{S} .
$$

where $\mathcal{A}(b, h, t)$ is the area of the isopycnal within a distance $h$ of the boundary and $d \mathcal{S}$ is an area element on that isopycnal. We then make use of the mathematical identity (Marshall et al. 1999)

$$
\frac{d}{d b} \iiint_{\mathcal{R}(b, h, t)} a(\mathbf{x}, t) d V=\iint_{\mathcal{A}(b, h, t)} \frac{a(\mathbf{x}, t)}{|\nabla b|} d \mathcal{S},
$$

for any field $a(\mathbf{x}, t)$, where $\mathcal{R}(b, h, t)$ corresponds to the region where buoyancy is less than $b$ below the height $h$. This identity allows the division by the potentially problematic buoyancy gradient magnitude $|\nabla b|$ to be replaced in favor of a buoyancy derivative. Applied to Eq. (8) this yields

$$
\mathcal{E}(b, h, t)=-\frac{d}{d b} \iiint_{\mathcal{R}(b, h, t)} \nabla \cdot \mathcal{B} d V
$$

Using the divergence theorem and the insulating boundary condition for $\mathcal{B}$ at the boundary then yields

$$
\mathcal{E}(b, h, t)=-\frac{d \mathcal{B}^{h}}{d b}-\frac{d}{d b}\left(\iiint_{\mathcal{A}(b, h, t)} \mathcal{B} \cdot \hat{\mathbf{n}} d \mathcal{S}\right),
$$

where

$$
d \mathcal{B}^{h}=\int_{d A} \mathcal{B} \cdot \hat{\mathbf{n}} d \mathcal{S}
$$

is the area-integrated turbulent buoyancy flux exiting the infinitesimal isopycnal layer across the height $z=h$ (where the normal vector $\hat{\mathbf{n}}$ points outward away from the region $\mathcal{R}$ ). By introducing the (positive) area-integrated turbulent buoyancy flux over the $b$ isopycnal,

$$
F(b, h, t)=-\iint_{\mathcal{A}(b, h, t)} \mathcal{B} \cdot \hat{\mathbf{n}} d \mathcal{S},
$$

and noting that along the $d A$ surface $d \mathcal{S} / d b=d x / b_{y}$, Eq. (11) can be rewritten as

$$
\mathcal{E}(b, h, t)=-\left.\int \frac{\mathcal{B}^{z}}{b_{y}}\right|_{z=h} d x+\frac{d F(b, h, t)}{d b},
$$

where the integral in the along-slope $x$ direction is performed along the $b$ isopycnal at the height $z=h$ and $\mathcal{B}^{z}$ is the slopenormal component of the buoyancy flux per unit area. An alternative derivation of Eq. (14), following the same approach as MF17, is provided in appendix A. Equation (14) holds generally for any isopycnal at any height $h$ even in the presence of unsteadiness.

When the turbulent buoyancy flux per unit area $\mathcal{B}$ is directed perpendicular to isopycnals (e.g., a diffusive buoyancy flux), then the first term in Eq. (14) will always be positive if the fluid is stably stratified at the height $z=h$ (i.e., $\mathcal{B}^{z}=-\kappa b_{z}$ is negative if $b_{z}$ is positive, while $b_{y}$ will always be positive at the last point at which the isopycnal crosses the height $h$ before encountering the boundary given a large-scale stable background stratification). Hence, our intuition that net upwelling should occur near the boundary will hold providing that upslope variations in the turbulent buoyancy flux across isopycnals between the height $h$ and the boundary [the second term in Eq. (14)] do not overwhelm the convergence of the turbulent buoyancy flux in the slope-normal direction. This is clearly true 
for small $h$ where $F(b, h, t)$ is small, while for larger $h$ the rate at which $F(b, h, t)$ must vary with $b$ in the upslope direction to achieve net downwelling turns out to be quite large, as examined in section 5. This second term in Eq. (14) is the abyssal ocean analog of water-mass transformation driven by the convergence of the lateral diffusive buoyancy flux across near vertical isopycnals in the mixed layer (Marshall et al. 1999). To continue further, we examine the diapycnal velocities and transport that arises in simple solutions taken from onedimensional boundary layer theory.

\section{Diapycnal transport in a stratified one-dimensional boundary layer}

\section{a. One-dimensional boundary layer theory}

We consider the flow near an abyssal boundary with constant slope and far-field interior stratification $N^{2}$ such that

$$
\begin{aligned}
& \frac{\partial b}{\partial z} \rightarrow N^{2} \cos \theta, \quad \text { as } \quad z \rightarrow \infty, \\
& \frac{\partial b}{\partial y}=N^{2} \sin \theta .
\end{aligned}
$$

Throughout most of the article we will use a bottom-intensified diffusivity with exponential form,

$$
\kappa(z)=\kappa_{\infty}+\left(\kappa_{0}-\kappa_{\infty}\right) e^{-z / d},
$$

with far-field value $\kappa_{\infty}$, near boundary value $\kappa_{0}$ a decay scale $\hat{d}$. However, in section $3 \mathrm{e}$ we briefly discuss the sensitivity of our results to other choices of the form for the diffusivity in the BBL.

An approximate one-dimensional (uniform in the upslope and along-slope directions) solution to the steady, rotating equations of motion (e.g., Garrett et al. 1993), discussed in more detail in Holmes et al. (2019) and Callies (2018), can be derived assuming that the decay scale $\hat{d}$ of the diffusivity is much larger than the thickness scale $q_{0}^{-1}$ of the layer in which friction is important in the constant $\kappa$ case,

$$
q_{0}^{4}=\frac{N^{2} \sin ^{2} \theta}{4 \operatorname{Pr}_{v 0} \kappa_{0}^{2}}\left[1+\left(\operatorname{SPr}_{u 0}\right)^{-1}\right]
$$

In Eq. (18), $S=N^{2} \tan ^{2} \theta / f^{2}$ is the slope Burger number, $f$ is the Coriolis parameter and $\operatorname{Pr}_{u 0}=\nu_{u 0} / \kappa_{0}$ and $\operatorname{Pr}_{v 0}=\nu_{v 0} / \kappa_{0}$ are the Prandtl numbers near the boundary associated with the viscosity in the along-slope $(x)$ and upslope $(y)$ momentum equations, respectively. The approximate analytic solution is given by (see appendix A of Holmes et al. 2019),

$$
\begin{aligned}
\Psi(z)= & \frac{\cot \theta}{1+\left(\operatorname{SPr}_{u 0}\right)^{-1}}\left[\kappa+\left(\operatorname{SPr}_{u 0}\right)^{-1} \kappa_{\infty}\right] \\
& {\left[1-e^{-q_{0} z}\left(\cos q_{0} z+\sin q_{0} z\right)\right], } \\
\frac{\partial b}{\partial z}= & \frac{N^{2} \cos \theta}{1+\left(\operatorname{SPr}_{u 0}\right)^{-1}}\left[1+\left(S \operatorname{Pr}_{u 0}\right)^{-1} \frac{\kappa_{\infty}}{\kappa}\right] \\
& {\left[1-e^{-q_{0} z}\left(\cos q_{0} z+\sin q_{0} z\right)\right], }
\end{aligned}
$$

where $\Psi(z)$ is the net upslope transport (per along-slope meter) below the slope-normal height $z$ such that the upslope velocity $V=\partial \Psi / \partial z$. In steady state $\Psi(z)$ is equivalent to the net diapycnal transport below the height $z$, or indeed the net transport across a horizontal surface between the height $z$ and the boundary (Garrett et al. 1993; Garrett 2001).

As highlighted by Callies (2018), for typical abyssal ocean parameters and order one Prandtl number this solution predicts unrealistically weak stratification throughout the SML where $\kappa$ is enhanced [Eq. (20)]. This weak stratification is due to the lack of representation of baroclinic-instability-driven restratification. A realistic stratification can be recovered using a large $\operatorname{Pr}_{u 0}$, physically interpreted as a parameterization for restratification by baroclinic eddies based on the thickness-weighted average formalism (Rhines and Young 1982; Greatbatch and Lamb 1990). Here, as we will mainly discuss the flow within the BBL, we will focus on the limit $S \operatorname{Pr}_{u 0} \rightarrow \infty$ where eddies maintain the stratification in the SML at its far-field value $N^{2}$. However, in section $3 \mathrm{~d}$ we briefly discuss the impact of reduced SML stratification through a finite choice for $\operatorname{Pr}_{u 0}$.

Example profiles of the buoyancy gradient [Eq. (20)], the buoyancy flux and the various diapycnal velocity components in the limit $S \operatorname{Pr}_{u 0} \rightarrow \infty$ are shown in Fig. 2 for the parameters $\tan \theta=1 / 100, N^{2}=10^{-6} \mathrm{~s}^{-1}, \kappa_{\infty}=10^{-5} \mathrm{~m}^{2} \mathrm{~s}^{-1}, \kappa_{0}=10^{-3} \mathrm{~m}^{2} \mathrm{~s}^{-1}$, $\hat{d}=200 \mathrm{~m}, \operatorname{Pr}_{v 0}=1, S \operatorname{Pr}_{u 0} \rightarrow \infty$. While these solutions do not capture the full complexity present in more realistic BBLs (e.g., Allen and Newberger 1998; Umlauf and Burchard 2011; Winters 2015; van Haren 2017; Wenegrat et al. 2018; Ruan et al. 2019), they serve as a useful starting point.

\section{b. Contributions to diapycnal flow within the $B B L$}

The slope-normal buoyancy gradient from the one-dimensional solutions [Eq. (20) in the limit $\operatorname{SPr}_{u 0} \rightarrow \infty$ ] shows a maximum at $z=\pi q_{0}^{-1}$, slightly increased from its far-field value of $N^{2}$, before decaying to zero as the boundary is approached (blue line in Fig. 2a, where the dashed black line marks $z=\pi q_{0}^{-1}$ ). The magnitude of the slope-normal component of the buoyancy flux $\kappa b_{z}$, whose divergence determines the diapycnal velocity in the one-dimensional case [Eq. (3) yields $\omega=\partial_{z}\left(\kappa b_{z}\right) /|\nabla b|$ ], shows a similar structure with the additional effect of the gradual reduction in $\kappa$ over its 200-m decay scale (green line in Fig. 2a). The strong convergence of the slope-normal component of the buoyancy flux $\kappa b_{z}$ drives diapycnal upwelling (blue line in Figs. 2b,c) which transitions to diapycnal downwelling away from the boundary at the top of what we define as the BBL (where $\omega=0$, solid black line in Fig. 2). This highlights the fact that much of the BBL must be well stratified, as the top of the BBL corresponds to the maximum in the buoyancy flux and therefore cannot have a stratification much reduced from that in the SML.

While the slope-normal component of the diffusive buoyancy flux goes to zero at the boundary there is still a small but nonzero upslope component $-\kappa b_{y}$. This means that the magnitude of the buoyancy flux $\mathcal{B}=\kappa|\nabla b|$ is not actually zero at the boundary (although it is too small to distinguish from zero, orange dashed line in Fig. 2a). However, in this onedimensional context the upslope component of the buoyancy 

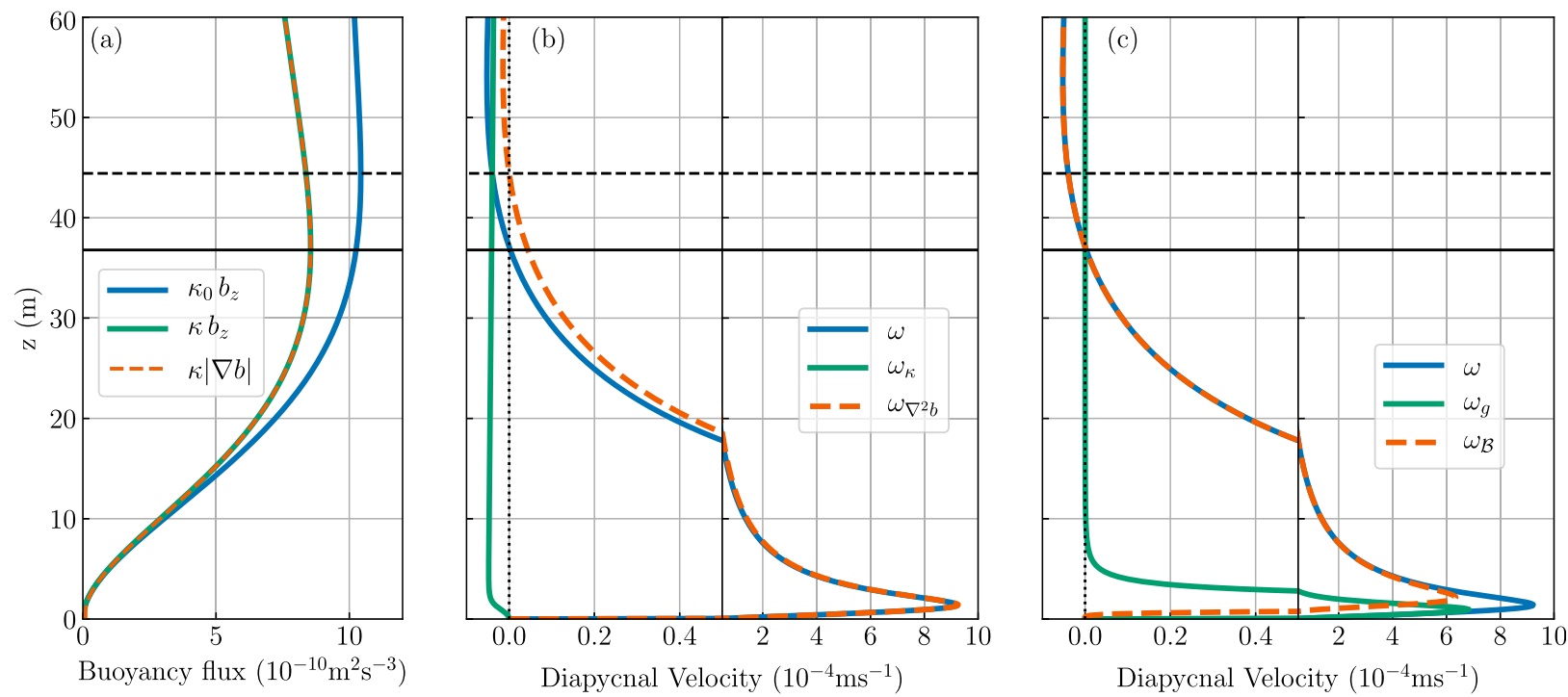

FIG. 2. Profiles from one-dimensional boundary layer theory. (a) The magnitude of the turbulent buoyancy flux in the $z$ direction $\kappa b_{z}$, the magnitude of the turbulent buoyancy flux $\mathcal{B}=\kappa|\nabla b|$, and the slope-normal buoyancy gradient multiplied by the near-bottom diffusivity $\kappa_{0} b_{z}$. (b),(c) Various contributions to the total diapycnal velocity $\omega$. Note that the $x$ scale has been split into two sections. The parameters used are a slope of $1 / 100, N^{2}=10^{-6} \mathrm{~s}^{-2}, \kappa_{\infty}=10^{-5} \mathrm{~m}^{2} \mathrm{~s}^{-1}, \kappa_{0}=10^{-3} \mathrm{~m}^{2} \mathrm{~s}^{-1}$, and $\hat{d}=200 \mathrm{~m}$. The solid black line marks the top of the BBL where $\omega=0$, and the dashed black line marks the height $z=\pi q_{0}^{-1}$. Note that the $y$ axis here is $z$ and does not correspond to the distance along an isopycnal, meaning that the relative contribution of different components of the diapycnal velocity to the total diapycnal transport cannot be assessed from these profiles (see Fig. 3).

flux has no convergence, meaning that $\omega$ is zero at the boundary. If upslope variability were permitted, then $\omega$ could be nonzero at the boundary (although in a steady solution $\omega$ must be zero at the boundary to satisfy a no-slip boundary condition). However, as discussed in more detail in section 5, upwelling would still be expected to dominate in the BBL due to the difference in length scales between along-boundary and slope-normal gradients.

The BBL upwelling is strongly peaked in the lower portion of the $\mathrm{BBL}$ where the convergence of the slope-normal buoyancy flux is maximum. This structure is a consequence of the structure in $b_{z}$, with $\omega$ closely following the term $\omega_{\nabla^{2} b}$ (orange dashed line in Fig. 2b). The term $\omega_{\nabla^{2} b}$ is positive below the height $\pi q_{0}^{-1}$ (dashed black line in Fig. 2). If $\kappa$ were constant then this height would correspond to the top of the BBL where $\omega=0$. Instead, with a bottom-intensified diffusivity, $\omega_{\kappa}$ is negative (green line in Fig. 2b) shifting the top of the BBL (and the height of maximum upslope transport $\Psi$ ) toward the boundary. The $\omega_{\kappa}$ drives weak but extensive downwelling except close to the boundary where the isopycnal normal points along the boundary $\hat{\mathbf{n}}=\hat{\mathbf{y}}$ in which direction $\kappa$ is constant [Eq. (6)]. Therefore, in the one-dimensional solutions (and assuming no reduction in SML stratification), $\omega_{\kappa}$ captures the "SML downwelling" referred to by MF17 and Holmes et al. $(2018,2019)$, while $\omega_{\nabla^{2} b}$ captures the BBL upwelling. However, $\omega_{\nabla^{2} b}$ also contributes a small amount of downwelling above $z=\pi q_{0}^{-1}$ because of the slight enhancement of stratification there.

The diapycnal transport can also be split into components arising from variations in the buoyancy gradient magnitude $\omega_{\mathcal{B}}$ and isopycnal curvature $\omega_{g}$ (orange dashed and green solid lines, respectively, in Fig. 2c). The buoyancy gradient magnitude $\omega_{\mathcal{B}}$ dominates throughout most of the BBL. However, in the lower portion of the BBL where isopycnal curvature is maximum $\omega_{g}$ also contributes. The isopycnal curvature drives upwelling here because isopycnals must curve downward (on average) to encounter the boundary at right angles and thus are concave with respect to the positive buoyancy coordinate. For small slopes, this term is only significant close to the boundary in the lowest part of the $\mathrm{BBL}$ as it is only here that the isopycnals depart significantly from the horizontal.

\section{c. Contributions to net upwelling across the BBL}

Figure $2 \mathrm{c}$ suggests that $\omega_{g}$ may make a contribution to net diapycnal upwelling within the BBL. However, $\omega$ is defined as the diapycnal transport per unit isopycnal area, not per unit height $z$. For the 1D solutions where gradients are constant in $y$, the net diapycnal transport [Eq. (8)] associated with a particular diapycnal velocity component $\omega_{c}$ within a distance $h$ of the boundary can be written in terms of an integral in the slopenormal coordinate $z$ (also see Garrett 2001),

$$
\begin{aligned}
\mathcal{E}_{c}(h) & =\iint_{0}^{h} \omega_{c} \frac{d s}{d z} d z d x, \\
& =\iint_{0}^{h} \omega_{c} \frac{|\nabla b|}{b_{y}} d z d x,
\end{aligned}
$$

where $d s$ is a distance along the isopycnal which has been rewritten in terms of the buoyancy gradient magnitude as $d s / d z=|\nabla b| / b_{y}$. This metric term converts diapycnal transport per unit area of an isopycnal to diapycnal transport per unit $z$. 
For the steady one-dimensional solutions a diapycnal transport per unit $z$ is equivalent to an upslope velocity $V_{c}$ (i.e., $\omega_{c}|\nabla b|=$ $V_{c} b_{y}$ ), or equivalently the diapycnal transport below the height $h$ is equivalent to the upslope transport below the height $h\left[\mathcal{E}_{c}(h)=\int \Psi_{c}(h) d x\right]$.

For the full diapycnal velocity $\omega_{c}=\omega$ [Eq. (3)], Eq. (22) reduces to the expression derived in section 2 c for $\mathcal{E}(h)$ [Eq. (14)], where $d F / d b=0$ for the one-dimensional solutions. By definition $\mathcal{E}(h)$ peaks at the top of the BBL where $\omega=0$. Much of this BBL upwelling is associated with flow in the lower portion of the BBL (Fig. 2c). In fact, in the case of a constant diffusivity it can be shown that the bottom $\pi^{-1}$ of the BBL hosts half of the net upwelling (see appendix B).

Integrating through the SML into the far-field yields the net diapycnal transport,

$$
\mathcal{E} \rightarrow \mathcal{E}^{\infty}=\kappa_{\infty} \cot \theta \int d x, \quad \text { as } \quad h \rightarrow \infty .
$$

This transport, associated with the far-field diffusivity, is small in this one-dimensional context. Similar expressions can be derived for the net diapycnal transports due to the individual contributions to $\mathcal{E}^{\infty}$ (see appendix C). In the limit $q_{0}^{-1} \ll \hat{d}$ these reduce to

$$
\begin{aligned}
\mathcal{E}_{\kappa}^{\infty} & \approx \cot \theta\left(\kappa_{\infty}-\kappa_{0}\right) \int d x, \\
\mathcal{E}_{\nabla^{2} b}^{\infty} & \approx \kappa_{0} \cot \theta \int d x, \\
\mathcal{E}_{g}^{\infty} & \approx \kappa_{0} \arctan (\cot \theta) \int d x,
\end{aligned}
$$

with $\mathcal{E}_{\mathcal{B}}^{\infty}=\mathcal{E}^{\infty}-\mathcal{E}_{g}^{\infty}$. As $\mathcal{E}_{\nabla^{2} b}^{\infty}$ corresponds to the total BBL upwelling in the one-dimensional solution, the "amplification factor" quantifying the ratio of BBL upwelling to total upwelling in this solution is given by

$$
\frac{\mathcal{E}_{\nabla^{2} b}^{\infty}}{\mathcal{E}^{\infty}}=\frac{\kappa_{0}}{\kappa_{\infty}}
$$

This can be large, but only because the net transport in the onedimensional solution is small.

More interesting is the fraction of BBL upwelling associated with isopycnal curvature,

$$
\frac{\mathcal{E}_{g}^{\infty}}{\mathcal{E}_{\nabla^{2} b}^{\infty}} \approx \frac{\kappa_{0} \arctan (\cot \theta)}{\kappa_{0} \cot \theta}=\tan \theta \arctan (\cot \theta)=\left(\frac{\pi}{2}-\theta\right) \tan \theta
$$

This function is plotted in Fig. 3a as a function of the slope $\tan \theta$. Counterintuitively, the contribution of isopycnal curvature to BBL upwelling increases as the slope increases even though the isopycnal slope experiences less change approaching a steep slope than a shallow slope. This is because the transport due to isopycnal curvature is relatively constant at slopes less than $1 / 10\left[\arctan \left(x^{-1}\right)\right.$ does not change much for small $x$ ], while the BBL transport $\mathcal{E}_{\nabla^{2} b}^{\infty}$ decreases significantly as the slope increases [Eq. (25)]. Profiles of the contributions to net BBL
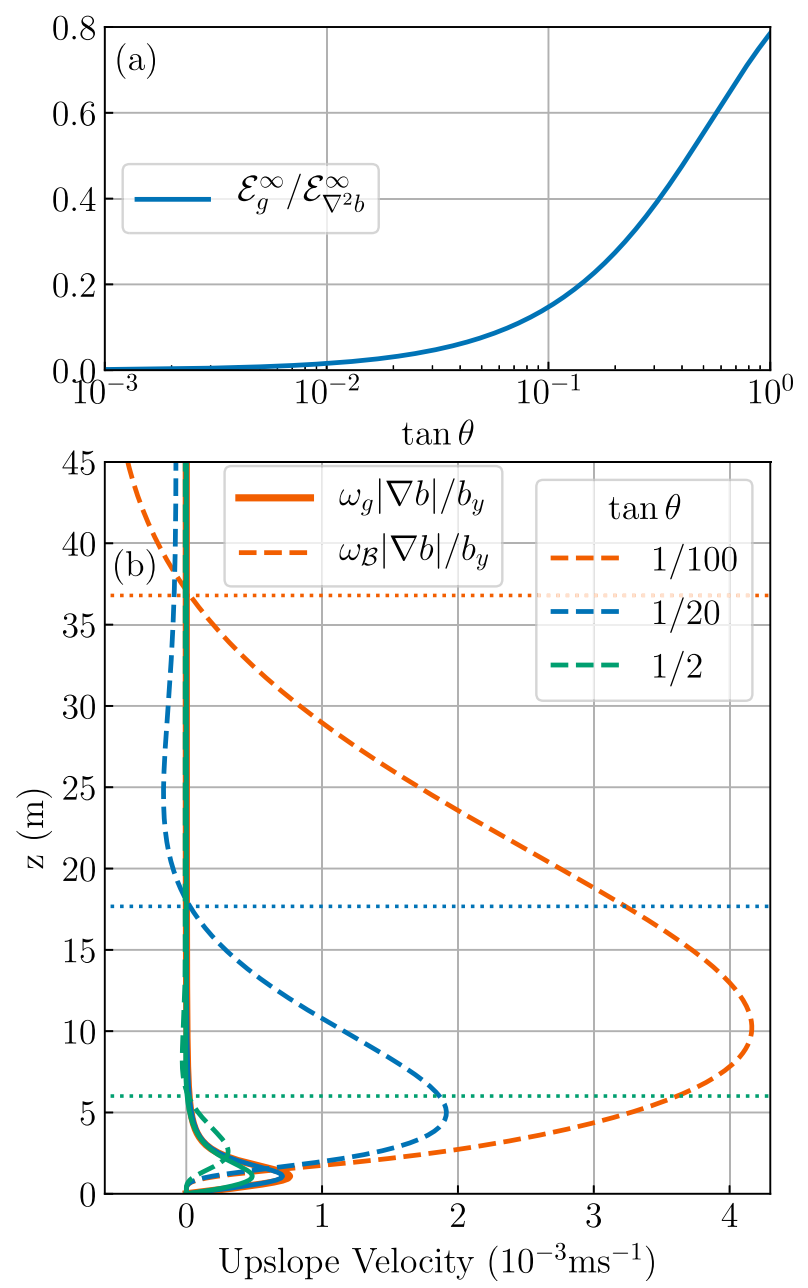

FIG. 3. Structure and properties of the isopycnal curvature contribution to diapycnal transport $\omega_{g}$ in the one-dimensional boundary layer solutions. (a) The fraction of diapycnal upwelling in the BBL due to isopycnal curvature $\mathcal{E}_{g}^{\infty} / \mathcal{E}_{\nabla^{2} b}^{\infty}=\tan \theta \arctan (\cot \theta)$ as a function of boundary slope $\tan \theta$. (b) The upslope velocity, or diapycnal transport per unit $x$ distance along-slope and per unit $z$ (in contrast to Fig. 2 where the diapycnal velocity is per unit area of an isopycnal) for slopes of $\tan \theta=1 / 100$ (orange lines), $\tan \theta=1 / 20$ (blue lines), and $\tan \theta=1 / 2$ (green lines) associated with isopycnal curvature $\omega_{g}$ (solid lines) and the buoyancy flux magnitude $\omega_{\mathcal{B}}$ (dashed lines). The top of the BBL is shown in dotted lines.

upwelling $\left(\omega_{g}\right.$ and $\omega_{\mathcal{B}}$, which sum to give $\omega_{\nabla^{2} b}$ in the limit $q_{0}^{-1} \ll \hat{d}$ ) which include the metric term $|\nabla b| / b_{y}$ indeed show that the BBL transport decreases as the slope increases, while the isopycnal curvature term remains relatively constant (Fig. 3b). Thus, for small slopes the geometry term does not make a significant contribution to the total diapycnal transport. However, it may be significant or even dominant for steep slopes, such as the sides of fracture zone canyons thought to host much of the mixing that converts Antarctic bottom water back to lighter waters (Thurnherr et al. 2020). For example, the northern wall of the fracture zone canyon shown in Fig. 5 of Thurnherr et al. (2020) has a very steep slope of 

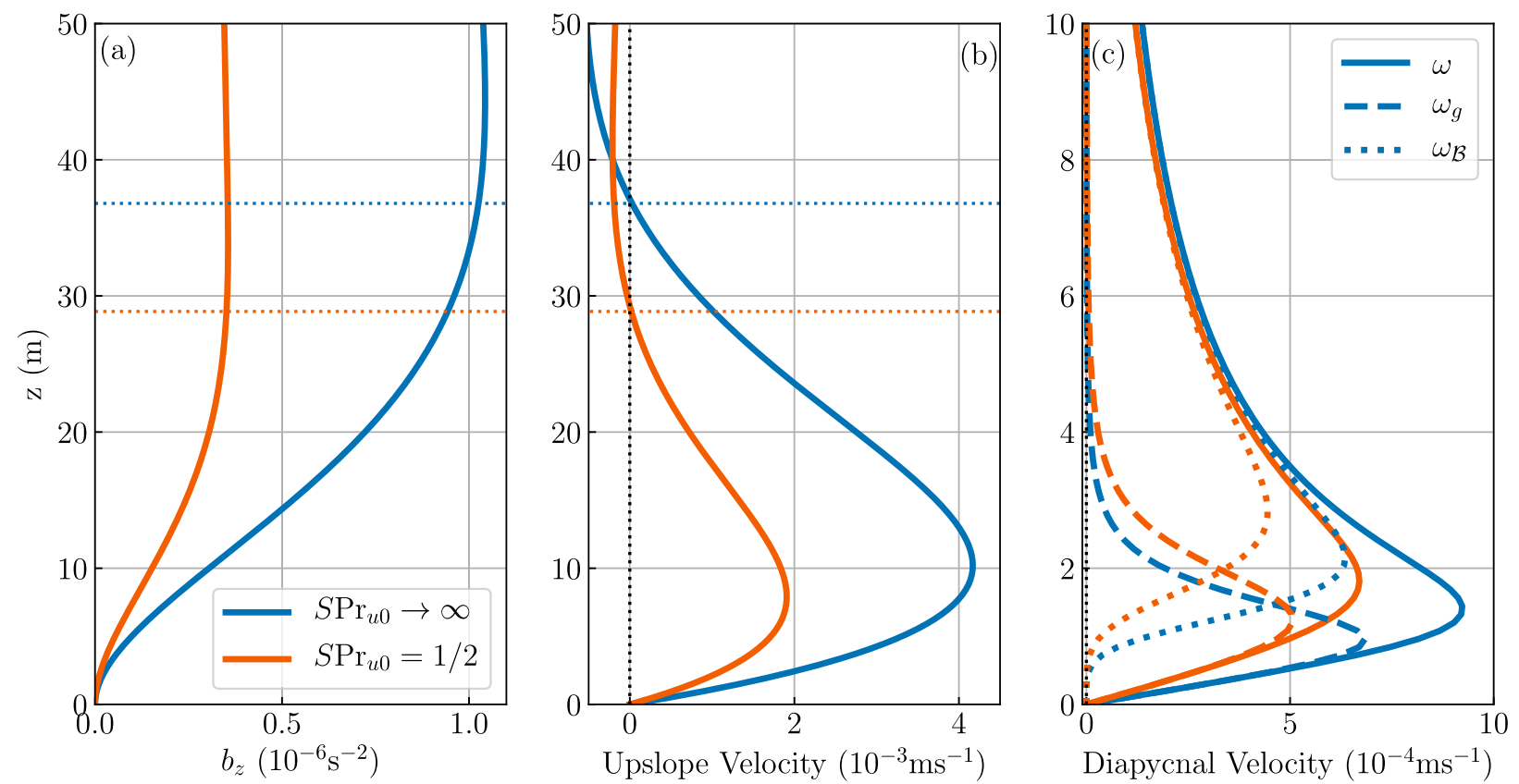

FIG. 4. A comparison of the (a) slope-normal buoyancy gradient $b_{z}$, (b) upslope velocity, and (c) diapycnal velocity between solutions in the limit $S \operatorname{Pr}_{u 0} \rightarrow \infty$ (as used elsewhere in the article, blue) and with a Prandtl number chosen such that the SML stratification is reduced by a factor of 3. Panel (c) (note the reduced vertical scale to focus on the near boundary region) also shows the components of the diapycnal velocity arising from changes in isopycnal curvature (dashed lines) and buoyancy flux magnitude (dotted lines).

roughly $1 / 2$. For this slope the isopycnal curvature term accounts for more than $70 \%$ of the diapycnal upwelling in the BBL (Fig. 3a). This is because for such steep slopes the overall BBL transport, associated with the convergence of the slopenormal buoyancy flux, is much weaker. In terms of the decomposition of $\omega$ into $\omega_{\mathcal{B}}$ and $\omega_{g}$ in Eq. (4), the magnitude of the buoyancy flux at the boundary (associated with only the along-boundary component) is not significantly less than that in the interior, meaning that $\omega_{\mathcal{B}}$ (through $\left.\nabla \mathcal{B}\right)$ is much weaker (green dashed line in Fig. 3b).

\section{d. The impact of reduced SML stratification}

The solutions discussed above assume that isopycnals remain flat in the SML. However, observations from the Brazil Basin instead show that the stratification in the SML is reduced by a factor $\sim 3$ relative to the far-field (Callies 2018). To evaluate the impact of reduced SML stratification and sloping isopycnals on the diapycnal transport in the $\mathrm{BBL}$ we therefore compare a solution with the choice $S \operatorname{Pr}_{u 0}=1 / 2$ to the limit $S \operatorname{Pr}_{u 0} \rightarrow \infty$. Such a solution has a factor of 3 reduction in the slope-normal buoyancy gradient in the SML [Eq. (20)] along with a reduced BBL thickness by a factor $\sim 3^{-1 / 4} \approx 0.76[\mathrm{Eq}$. (18), Fig. 4a]. The net diapycnal or upslope transport within the $\mathrm{BBL}$ is reduced by a similar factor of 3 [Eq. (19), the area under the curve of Fig. 4b]. Throughout most of the BBL the diapycnal velocity and its various components show little change as the reduction in the convergence of the buoyancy flux associated with the reduction in $b_{z}$ is compensated by the factor $|\nabla b|^{-1}$ in Eq. (3) (Fig. 4c). However, in the bottom $\sim 5 \mathrm{~m}$ the diapycnal velocity (and its contributions from both $\omega_{g}$ and $\omega_{\mathcal{B}}$, dotted and dashed lines in Fig. 4c) does show a reduction due to the fact that the upslope component of the buoyancy gradient $b_{y}$, which does not depend on $S \operatorname{Pr}_{u 0}$, makes a significant contribution to $|\nabla b|$ [which itself is in the denominator of Eq. (3)]. We conclude that while eddy-driven restratification, associated in the one-dimensional solutions with the parameter $\operatorname{Pr}_{u 0}$, is an important process that influences the net diapycnal transport in the BBL and SML, the BBL thickness and the slope of isopycnals in the SML, with respect to the details of the flow in the BBL it acts largely as an overall scaling.

\section{e. The impact of the BBL diffusivity structure}

The one-dimensional boundary layer solutions discussed above assume a specific profile for the turbulent diffusivity that is largely constant within the BBL [Eq. (17), which will be referred to as $\kappa_{\exp }$ in this section, blue line in Fig. 5a]. In contrast, frictional boundary layer theory suggests that the turbulent diffusivity and viscosity should reduce linearly toward zero within a frictional log layer of thickness estimated as ranging from a few centimeters to a few meters (e.g., Wüest and Lorke 2003; Holtappels and Lorke 2011). In this section we examine the sensitivity of our results to an alternative choice for the turbulent diffusivity profile in the BBL where the diffusivity decays toward zero within the bottom $\sim 2 \mathrm{~m}$ of the BBL,

$$
\kappa_{\mathrm{dec}}=\kappa_{\exp }\left(1-\kappa_{m} e^{-z / z_{m}}\right),
$$

where $\kappa_{m}=0.95$ and $z_{m}=1 \mathrm{~m}\left(\kappa_{\mathrm{dec} 1}\right.$, orange line in Fig. 5). For completeness, we also consider a case with $z_{m}=10 \mathrm{~m}$ where the diffusivity decays over a length scale more comparable to 

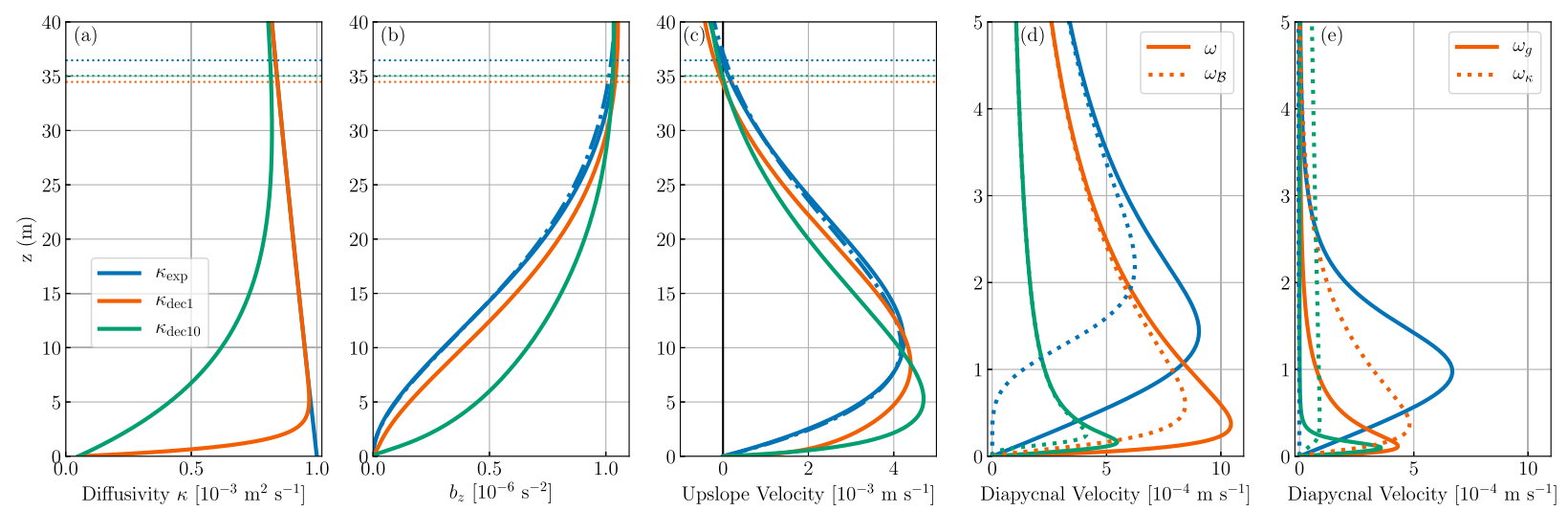

FIG. 5. A comparison of the BBL structure between 1D boundary layer solutions using two forms for the diffusivity in the boundary layer, an exponential decay (blue lines as used elsewhere in the article), and a simple representation of a frictional log layer where the diffusivity decays toward zero within either $\sim 2 \mathrm{~m}$ (orange lines) or $\sim 20 \mathrm{~m}$ (green lines) of the boundary [see Eq. (29) where $z_{m}=1 \mathrm{~m}$ for $\kappa_{\mathrm{dec} 1}$ or $z_{m}=10 \mathrm{~m}$ for $\kappa_{\mathrm{dec} 10}$ ]. Otherwise parameters are the same as used in Fig. 2. Shown are the (a) diffusivity, (b) slope-normal buoyancy gradient $b_{z}$, (c) upslope velocity, and (d),(e) diapycnal velocity and its components from numerical solutions to the onedimensional boundary layer equations [note the differing vertical scale for (d) and (e)]. The thin dotted lines in (a)-(c) mark the top of the BBL. The approximate analytic solution in the exponential diffusivity case used elsewhere in the article is also shown in (b) and (c) (dotdashed lines, see section 3a).

the BBL thickness $\left(\kappa_{\text {dec10 }}\right.$, green line in Fig. 5). Equation (29) does not satisfy the criteria needed for the approximate analytic solution [Eqs. (19), (20)] and thus we must resort to numerical solutions of the boundary layer equations. Following Callies (2018) we solve the one-dimensional steady-state boundary layer equations numerically by projecting them onto 2048 Chebyshev polynomials using the software package Dedalus (Burns et al. 2020). In the exponential diffusivity case we note that the numerical solution closely matches the approximate analytic solution used elsewhere in the article (cf. blue solid and dot-dashed lines in Figs. 5b,c). Parameters used here are identical to those used in Fig. 2.

The slope-normal buoyancy gradient is slightly enhanced for the $\kappa_{\mathrm{dec} 1}$ case throughout the BBL, which results in a shift in the upslope transport profile toward the boundary and a slight reduction in the BBL depth (cf. orange and blue lines in Figs. 5b,c). Upwelling transport is strongly enhanced in the bottom $\sim 2 \mathrm{~m}$ due to the convergence of the slope-normal buoyancy flux associated with the reduction in diffusivity. This has a knock-on effect throughout the rest of the BBL as the total upwelling transport across the BBL must remain the same. While overall these changes across the majority of the BBL are not large, the differences in the diffusivity near the boundary have a more dramatic influence on the diapycnal velocity, and its various components, within the bottom $5 \mathrm{~m}$ (Figs. 5d,e, note the focused vertical scale). The larger nearboundary slope-normal buoyancy gradient shifts the diapycnal velocity profile peak to within half a meter of the boundary (cf. solid orange and blue lines in Fig. 5d) and significantly reduces the contribution of the isopycnal curvature term $\omega_{g}$ (cf. solid orange and blue lines in Fig. 5e). The diapycnal velocity associated with changes in the buoyancy flux magnitude $\omega_{\mathcal{B}}$ now dominates the total diapycnal velocity (cf. orange dotted and solid lines in Fig. 5d). However, the convergence of the buoyancy flux associated with the decay in the diffusivity in the bottom $\sim 2 \mathrm{~m}$ now contributes significantly to the upward diapycnal velocity $\left(\omega_{\kappa}\right.$, orange dotted line in Fig. 5e), in contrast to the $\kappa_{\text {exp }}$ case where $\omega_{\kappa}$ drove a very weak downward diapycnal velocity throughout the BBL (e.g., green line in Fig. 2b). Increasing the length scale over which the diffusivity decays to a significant fraction of the BBL thickness results in a further increase in the BBL stratification, shifts the upslope velocity profile closer to the boundary and further decreases the magnitude of the diapycnal velocity (cf. green and orange lines in Fig. 5).

\section{Comparison with a well-mixed boundary layer}

In this section we compare the stratified diffusive onedimensional BBL to the fully mixed BBL structure of MF17. The isopycnal structure assumed in MF17 consists of an interior with constant stratification $N^{2}$ and flat isopycnals lying above a BBL where isopycnals are perpendicular to the boundary. In the slope-normal coordinate system this corresponds to

$$
\frac{\partial b^{\mathrm{MF} 17}}{\partial z}=\left\{\begin{array}{cc}
N^{2} \cos \theta, & \text { for } \quad z>H^{\mathrm{BBL}} \\
0, & \text { for } z<H^{\mathrm{BBL}}
\end{array}\right.
$$

where $b_{y}=N^{2} \sin \theta$, as in the one-dimensional solution. The BBL thickness $H^{\mathrm{BBL}}$, now an externally imposed parameter, is chosen to be the same as in the one-dimensional solution, corresponding once again to the change between diapycnal upwelling in the BBL and downwelling above. Visually comparing the isopycnal structure between the two solutions (Fig. 6a) emphasizes how stratified the one-dimensional solution is throughout most of the BBL. Consequently, the area of an isopycnal within the BBL is much larger in the one-dimensional solution than in the MF17 mixed layer.

To compare the diapycnal transport we will assume that at and above the height of the top of the BBL the diffusivity is the 

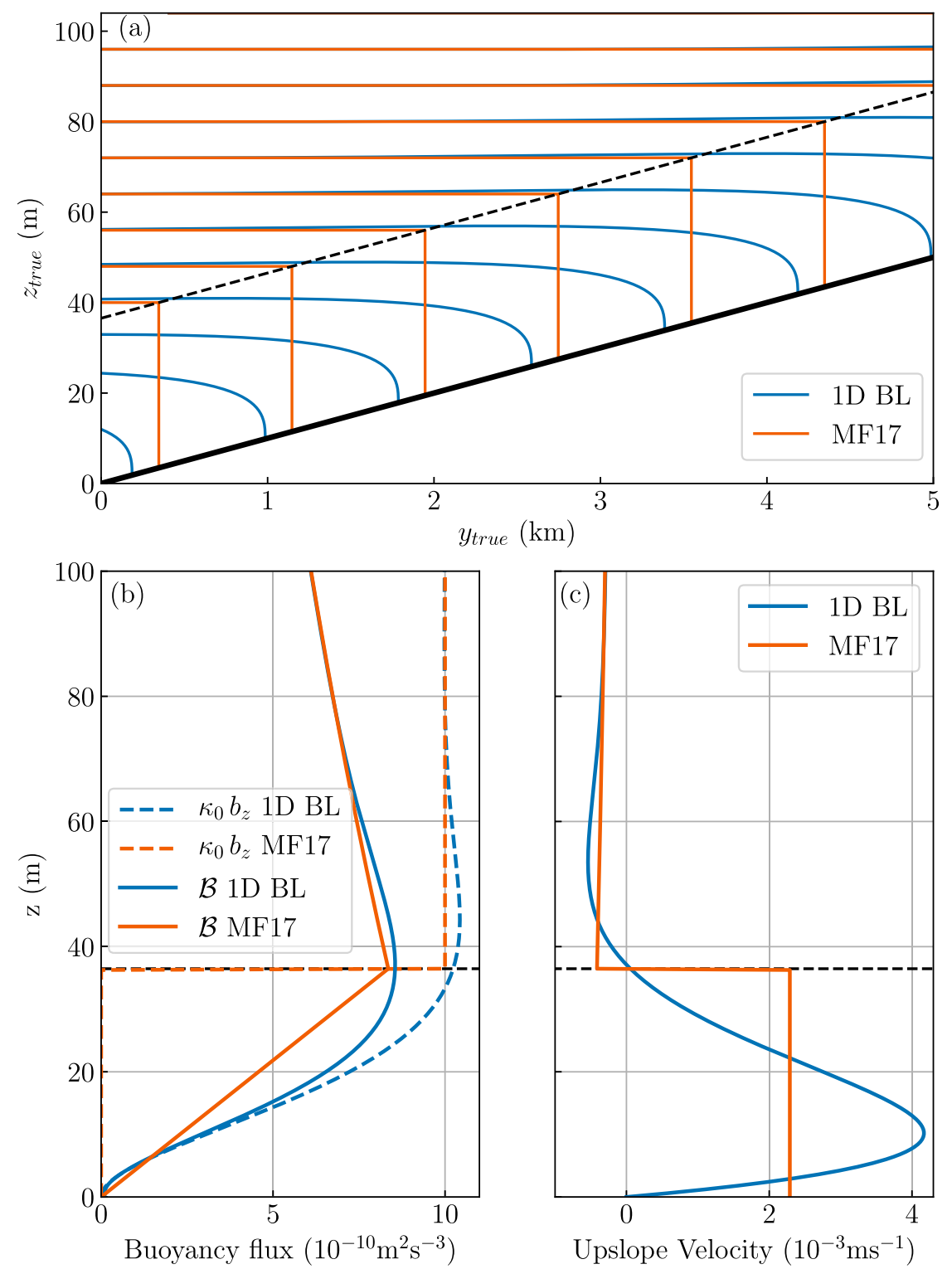

FIG. 6. A comparison of the BBL structure between the 1D boundary layer solution (blue lines) and the MF17 bottom mixed layer (MF17, orange lines) for a slope of 1/100. (a) Isopycnals, where the dashed black line indicates the top of the BBL. Note the highly exaggerated aspect ratio. (b) The magnitude of the turbulent buoyancy flux $\mathcal{B}$ (solid lines) and the buoyancy gradient multiplied by the near-bottom diffusivity $\kappa_{0} b_{z}$ (dashed lines). (c) The upslope velocity or diapycnal transport per unit $x$ and $z$.

same in the MF17 solution as it is in the one-dimensional boundary layer solution [Eq. (17)]. However, in the MF17 mixed layer the buoyancy flux is no longer related to the local buoyancy gradient. Instead, diapycnal transport is achieved by a nonlocal buoyancy flux in the slope-normal direction with magnitude $\mathcal{B}$ that is assumed to decay linearly through the BBL (orange line in Fig. 6b). The maximum in the buoyancy flux at the top of the BBL is determined by matching to the interior solution (i.e., the diffusivity at that height multiplied by the buoyancy gradient). This is slightly weaker than the peak buoyancy flux in the one-dimensional solution because of the small enhancement in $b_{z}$ at the top of the BBL in that solution (cf. dashed blue and orange lines in Fig. 6b).

While both solutions show diapycnal upwelling in the BBL and downwelling above, the structure of the transport is quite different (Fig. 6c). Diapycnal upwelling within the MF17 mixed layer is constant (as the linear buoyancy flux leads to uniform buoyancy gain), while in the $1 \mathrm{D}$ solution it is focused in the lower portion of the BBL. The diapycnal velocity $\omega$ (diapycnal transport per unit area of an isopycnal) in the MF17 
mixed layer is in fact much larger than the one-dimensional solution, due to the division by the small uniform upslope buoyancy gradient [Eq. (3)]. However, the area of an isopycnal within the MF17 mixed layer is much smaller than in the onedimensional solution, meaning that the net BBL diapycnal transport is similar between the two solutions (being determined solely by the maximum in the buoyancy flux at the top of the BBL).

Another notable feature of the MF17 mixed layer is that the transition between diapycnal upwelling and diapycnal downwelling at the top of the BBL is discontinuous. However, overall the structure of the diapycnal transport outside of the $\mathrm{BBL}$ is similar in the two solutions.

\section{Upslope variability}

The solutions discussed previously did not vary in the upslope direction. In those solutions, where there are no upslope variations in the buoyancy flux through the term $d F / d b$ in Eq. (14), diapycnal upwelling always occurs over some layer near the boundary due to the convergence of the slope-normal component of the buoyancy flux [first term in Eq. (14)]. How large would $d F / d b$ have to be to reverse the sign of $\mathcal{E}$ and therefore remove the upwelling? Upslope variations in $F$ can occur through variations in the turbulent diffusivity, in the buoyancy gradients or in topography. In this section we discuss this question by presenting a simple scaling argument where $F$ is assumed to vary over some characteristic upslope scale $\Delta y$, but otherwise the structure of the steady-state one-dimensional boundary layer solution is maintained.

For simplicity we will work with the one-dimensional solution where the diffusivity is assumed constant in the slope-normal direction. The area-integrated buoyancy flux along an isopycnal below the top of the BBL (i.e., where $z=H^{\mathrm{BBL}}=\pi q_{0}^{-1}$ ) is given by

$$
\begin{aligned}
F^{\mathrm{BBL}} & \equiv F\left(\pi q_{0}^{-1}\right)=\iint_{0}^{\pi q_{0}^{-1}} \kappa_{0}|\nabla b| \frac{|\nabla b|}{b_{y}} d z d x \\
& =\left\{\kappa_{0} N^{2} \cos \theta \cot \theta \int_{0}^{\pi q_{0}^{-1}}[1-r(z)]^{2} d z+\kappa_{0} \pi q_{0}^{-1} N^{2} \sin \theta\right\} \int d x
\end{aligned}
$$

$$
=\kappa_{0} N^{2} q_{0}^{-1} \sin \theta\left[\cot ^{2} \theta\left(\pi-2 e^{-\pi}-\frac{3}{4} e^{-2 \pi}-\frac{5}{3}\right)+\pi\right] \int d x,
$$

$$
\approx \kappa_{0} N^{2} \cos \theta \pi q_{0}^{-1} \cot \theta\left(1.4 \pi^{-1}+\tan ^{2} \theta\right) \int d x,
$$

where $r(z)=e^{-q_{0} z}\left(\sin q_{0} z+\cos q_{0} z\right)$ and the numeric factor inside the brackets multiplying $\cot ^{2} \theta$ in Eq. (33) is close to 1.4. Equation (34) reveals that $F^{\mathrm{BBL}}$ is equal to the slopenormal buoyancy flux per unit area at the top of the BBL, $\kappa_{0} N^{2} \cos \theta$, times a fraction of $\approx 1.4 \pi^{-1}\left(\tan ^{2} \theta\right.$ is small for small slopes) of the lateral area of the BBL, $\pi q_{0}^{-1} \cot \theta \int d x$. If $F^{\mathrm{BBL}}$ were to vary over a characteristic upslope distance of $\Delta y$ through, for example, the diffusivity $\kappa_{0}$ reducing to zero over that distance, then

$$
\frac{d F}{d b} \sim \frac{F^{\mathrm{BBL}}}{b_{y} \Delta y} \approx \frac{1.4 \kappa_{0} q_{0}^{-1} \cot ^{2} \theta}{\Delta y} \int d x,
$$

where we have dropped the small parameter $\tan ^{2} \theta$. To prevent any net diapycnal upwelling across the BBL, $\Delta y$ must be sufficiently small such that $d F / d b$ given by Eq. (35) is the same size as the first term in Eq. (14), yielding the requirement (where we ignore the slight increase in $b_{z}$ at the top of the boundary layer),

$$
\begin{aligned}
\frac{d F}{d b} & \sim-\left.\int \frac{\mathcal{B} \cdot \hat{\mathbf{z}}}{b_{y}}\right|_{z=\pi q_{0}^{-1}} d x \sim \kappa_{0} \cot \theta \int d x, \\
& \Rightarrow \Delta y \sim 1.4 q_{0}^{-1} \cot \theta
\end{aligned}
$$

That is, $F^{\mathrm{BBL}}$ would have to vary over a distance similar to half $\left(1.4 \pi^{-1} \approx 0.45\right)$ of the lateral thickness of the BBL $\pi q_{0}^{-1} \cot \theta$. For a slope of $\tan \theta=1 / 100$ and for the parameters in Fig. 2 where $q_{0}^{-1} \approx 14 \mathrm{~m}$, this corresponds to $2 \mathrm{~km}$. This is in fact an overestimate of $\Delta y$, as if $\kappa$ were to vary over this scale then the calculation of $F^{\mathrm{BBL}}$ in Eq. (34) is no longer valid as it assumes that $\kappa$ is constant over each isopycnal in the boundary layer. ${ }^{1}$ Such a rate of upslope variation in the time-averaged turbulent buoyancy flux, whether arising from variations in the buoyancy gradients or diffusivity, may be quite rapid if it must arise from a change in the large-scale characteristics of the turbulence (e.g., the topographic roughness or the background stratification).

A similar scaling argument to that in Eq. (37) can be performed for the mixed layer BBL of MF17. In this case, the buoyancy flux at the top of the BBL is similar but $F^{\mathrm{BBL}}$ is different. Within the MF17 BBL isopycnals are perpendicular to the boundary, and thus only the component of the buoyancy flux parallel to the boundary contributes to $F^{\mathrm{BBL}}$. This buoyancy flux per unit area is $\kappa_{0} b_{y}$, however, the area of an isopycnal in the MF17 BBL is much smaller, being $H^{\mathrm{BBL}} \int d x=\pi q_{0}^{-1} \int d x$. Therefore, for the MF17 BBL,

$$
F^{\mathrm{BBL}}=\pi q_{0}^{-1} \kappa_{0} N^{2} \sin \theta \int d x \quad \mathrm{MF} 17 \mathrm{BBL} .
$$

This is a factor of $\sim \tan ^{2} \theta$ smaller than $F^{\mathrm{BBL}}$ in the onedimensional boundary layer case [Eq. (34)]. Therefore, MF17 were correct to neglect the $F^{\mathrm{BBL}}$ term in the buoyancy budget given their assumed isopycnal geometry. However, their model of the BBL as a fully mixed layer may not be appropriate for the real ocean.

It should be noted that in the scaling calculations presented above such upslope variations in the turbulent buoyancy flux would create a significant convergence of the upwelling

\footnotetext{
${ }^{1}$ Alternatively, Eq. (34) would be correct if $\kappa$ was instead a function of $b$. However, in this case the downwelling associated with $d F^{\mathrm{BBL}} / d b$ would come from the strong dependence of $\kappa$ on $b$ and $z$ in the upper part of the BBL. Thus this region would effectively become part of the SML where the buoyancy flux decreases with height, rather than the BBL and thus the BBL would simply become narrower.
} 
diapycnal transport in the BBL, resulting in expulsion of fluid along isopycnals into the SML. Exchanges of fluid between the SML and BBL have been discussed by a number of authors (e.g., McPhee-Shaw 2006; Kunze et al. 2012; Winters 2015; Dell and Pratt 2015; Holmes et al. 2018) and would be expected to further alter the stratification and, potentially, the characteristics of the turbulence [e.g., Winters (2015) observe the expulsion of layers of high turbulence out of the boundary layer into the interior]. These changes would then be expected to feedback on the diapycnal transport in the BBL, but it remains unclear how such changes will manifest once approaching a steady state.

For the specific case of upslope variations in topography Dell and Pratt (2015) have shown that the dominance of upslope transport in the BBL can be overwhelmed for sufficiently large and rapid corrugations in a large-scale topographic slope. While they do not specifically discuss diapycnal transport, they do show that inertial overflows (where the near-bottom flow can be directed down the local bottom slope) can occur when the slope variations associated with corrugations are larger than the background topographic slope (e.g., their Fig. 11). However, once again these downslope flows are localized to lateral scales of order the horizontal scale of the boundary layer thickness and their solutions still show sustained upward transport when averaged over these corrugations.

\section{Discussion}

The one-dimensional boundary layer solutions examined in this article are idealized and smooth relative to turbulent nearbottom fields taken from highly sampled observations (e.g., van Haren 2017) or from high-resolution simulations (e.g., Winters 2015; Ruan et al. 2019). It is not yet clear whether the specific conclusions made above that depend on the structure of the one-dimensional solution would be expected to hold when averaging over this spatial and temporal complexity. Observations rarely show the reduction in the stratification (or dissipation rate) near the boundary that the one-dimensional solutions produce (e.g., van Haren 2017; Thurnherr et al. 2020), although there are some exceptions (e.g., Kunze et al. 2012). This seems to suggest that oceanic BBLs are often too thin to be resolved by existing microstructure measurements, highlighting the need for a future field campaign that can measure buoyancy fluxes and isopycnal structure from the $\sim 500$-m scale of the SML down to a distance of meters from the boundary over the range of time-scales present in the turbulent flow.

One important question that remains to be addressed is the nature of the averaging required to convert measurements made in a complex, time-dependent turbulent near bottom flow field into a sensible measure of diapycnal buoyancy and mass fluxes. Most studies take advantage of the identity in Eq. (9) to convert the area-integral of the diapycnal velocity [Eq. (8), which includes a potentially convoluted area integral as well as the problematic division by $|\nabla b|]$ to a more tractable volume integral of the buoyancy flux convergence over the volume bounded by two isopycnals [e.g., Eq. (10), Ferrari et al. 2016; de Lavergne et al. 2016]. In large-scale contexts information on the spatial structure of the diapycnal transport is then typically lost. In a more local context where the properties of the BBL turbulence itself are of interest, a mixed buoyancy-space physical-space diagnostic may be more useful. Such a diagnostic is obtained by retaining the dependence on height-above-bottom $h$ in Eq. (10) by integrating the buoyancy flux convergence only up to $h$, while still following the temporal and spatial variability of isopycnals below this height (as shown by the gray region in Fig. 1). Application of Eq. (10) to highly resolved simulations or near-bottom turbulence measurements (e.g., Winters 2015; van Haren 2017) could prove useful in investigating the spatial structure of nearbottom diapycnal transport.

\section{Summary}

In this article we have examined the structure of diapycnal upwelling within a series of idealized abyssal BBLs. Conclusions can be stated as follows:

1) Net diapycnal upwelling is favored adjacent to the boundary due to the strong convergence of the slope-normal buoyancy flux there [first term in Eq. (14)]. Only variations in the BBL buoyancy flux along the boundary [second term in Eq. (14)] over distances of order the lateral width of the BBL (or equivalently the vertical thickness of the BBL times the inverse boundary slope) seem able to prevent this upwelling.

2) In one-dimensional boundary layer theory the diffusive buoyancy flux must peak in well-stratified fluid (i.e., the stratification cannot be reduced much below that in the SML). This peak corresponds to the transition between boundary diapycnal upwelling and interior diapycnal downwelling and thus much of the layer experiencing diapycnal upwelling (defined in this article as the BBL) must be well stratified. This indicates that alternative definitions of the BBL based purely on the stratification (as commonly used in the literature) may not be useful for identifying transitions in diapycnal transport.

3) In addition to variations in the diffusive buoyancy flux magnitude, the curvature of isopycnals also contributes to diapycnal transport. In the one-dimensional solutions this curvature term is only significant close to the boundary where isopycnals are curved (Fig. 2) and is sensitive to variations in the diffusivity very close to the boundary (Fig. 5). It makes only a small contribution to net upwelling within the BBL for small slopes but may be significant near steep slopes such as the walls of fracture zone canyons (see Fig. 3).

4) The well-stratified one-dimensional BBL, in which diapycnal upwelling is strongest in the lower portion (where the stratification is weakest), contrasts with the mixed-BBL model of MF17 where diapycnal transport is uniform. The diapycnal velocity is larger in the mixed-BBL model of MF17, but the net diapycnal transport is similar as the area of an isopycnal is smaller.

Acknowledgments. We thank Jörn Callies and an anonymous reviewer for useful comments. We gratefully acknowledge support from the Australian Research Council through Grant FL150100090. 


\section{APPENDIX A}

An Alternative Derivation of Eq. (14)

This appendix contains an alternative derivation of Eq. (14) following the method of MF17. The volume budget for the region $\mathrm{d} \mathcal{R}(b, h, t)$ in Fig. 1 is

$$
\begin{aligned}
\frac{\partial}{\partial t} \iiint_{d \mathcal{R}(b, h, t)} d V= & \iint_{\mathcal{A}(b-d b, h, t)} \omega d \mathcal{S}-\iint_{\mathcal{A}(b, h, t)} \omega d \mathcal{S} \\
& -\iint_{d A(b, h, t)} w d \mathcal{S}
\end{aligned}
$$

where $w$ is the velocity in the positive slope-normal direction $z$. The buoyancy budget of the same region (following Eq. A2 of MF17) is

$$
\begin{aligned}
\frac{\partial}{\partial t} \iiint_{d \mathcal{R}(b, h, t)} b^{\prime} d V= & \iint_{\mathcal{A}(b-d b, h, t)} b^{\prime} \omega d \mathcal{S}-\iint_{\mathcal{A}(b, h, t)} b^{\prime} \omega d \mathcal{S}-\iint_{d A(b, h, t)} w b^{\prime} d \mathcal{S}, \\
& +\iint_{\mathcal{A}(b-d b, h, t)} \mathcal{B} \cdot \hat{\mathbf{n}} d \mathcal{S}-\iint_{\mathcal{A}(b, h, t)} \mathcal{B} \cdot \hat{\mathbf{n}} d \mathcal{S}-\iint_{d A(b, h, t)} \mathcal{B}^{z} d \mathcal{S},
\end{aligned}
$$

where here we have used $b^{\prime}$ to denote the three-dimensional buoyancy field as opposed to the specific buoyancy value $b$. The term $\hat{\mathbf{n}}$ is the isopycnal normal. Note that in the first and second terms on the RHS of Eq. (A2) $b^{\prime}$ can be taken out of the integral as it is equal to $b-d b$ and $b$, respectively. This is an advantage of using a control volume bounded by isopycnals which have a single buoyancy value. An alternative choice of control volume, bounded for example by vertical, horizontal or slope-normal lines on its upper and lower edge, would be more difficult to work with for this reason. Note that the second and third last terms in Eq. (A2) were of negligible magnitude when applied to the well-mixed BBL of MF17. Subtracting $b$ times Eq. (A1) from Eq. (A2) yields

$$
\begin{aligned}
\frac{\partial}{\partial t} \iiint_{d \mathcal{R}(b, h, t)}\left(b^{\prime}-b\right) d V= & -d b \iint_{\mathcal{A}(b-d b, h, t)} \omega d \mathcal{S}-\iint_{d A(b, h, t)} w\left(b^{\prime}-b\right) d \mathcal{S} \\
& +\iint_{\mathcal{A}(b-d b, h, t)} \mathcal{B} \cdot \hat{\mathbf{n}} d \mathcal{S}-\iint_{\mathcal{A}(b, h, t)} \mathcal{B} \cdot \hat{\mathbf{n}} d \mathcal{S}-\iint_{d A(b, h, t)} \mathcal{B}^{z} d \mathcal{S} .
\end{aligned}
$$

Dividing Eq. (A3) by $d b$, realizing that along the surface $d A(b$, $h, t), d \mathcal{S} / d b=d x / b_{y}$ and taking the limit $d b \rightarrow 0$ yields Eq. (14).

\section{APPENDIX B}

\section{The Average Height of BBL Upwelling}

Much of the diapycnal upwelling in the one-dimensional solution occurs in the bottom portion of the BBL (e.g., blue line in Fig. 3b). This can be evaluated more quantitatively by computing the diapycnal-transport weighted height defined by

$$
\bar{z} \equiv \frac{\int_{b} z \omega d s}{\int_{b} \omega d s} .
$$

For the case of a constant diffusivity this is easy to evaluate [using Eq. (20) in the limit $S \mathrm{Pr}_{u 0} \rightarrow \infty$ ],

$$
\begin{aligned}
\bar{z} & =\frac{\int_{0}^{\infty} z b_{z z} d z}{\int_{0}^{\infty} b_{z z} d z}, \\
& =2 q_{0} \int_{0}^{\infty} z e^{-q_{0} z} \sin q_{0} z d z, \\
& =q_{0}^{-1} .
\end{aligned}
$$

Thus, the bottom $\pi^{-1}$ of the BBL accounts for half the BBL transport. Note that this calculation also includes the small amount of diapycnal downwelling that arises in the constant diffusivity case because of the slight increase in $b_{z}$ at the top of the BBL.

\section{APPENDIX C}

\section{Mathematical Expressions for the Geometric Contributions to Diapycnal Transport in the One-Dimensional Solutions}

Expressions for the various geometric diapycnal velocity terms discussed in section $2 \mathrm{~b}$ in the $y-z$ coordinate system are

$$
\begin{aligned}
\omega_{|\nabla b|} & =\frac{\kappa}{|\nabla b|^{3}}\left(b_{z}^{2} b_{z z}+2 b_{y} b_{z} b_{y z}+b_{y}^{2} b_{y y}\right), \\
\omega_{\nabla^{2} b} & =\frac{\kappa}{|\nabla b|}\left(b_{z z}+b_{y y}\right), \\
\omega_{g} & =\frac{\kappa}{|\nabla b|^{3}}\left(b_{y}^{2} b_{z z}-2 b_{y} b_{z} b_{y z}+b_{z}^{2} b_{y y}\right), \\
\omega_{\kappa} & =\frac{\kappa_{z} b_{z}+\kappa_{y} b_{y}}{|\nabla b|},
\end{aligned}
$$

with $\omega_{\mathcal{B}}=\omega_{|\nabla b|}+\omega_{\kappa}$. In the one-dimensional solutions $\kappa_{y}, b_{y y}$, and $b_{y z}$ are all zero. 
Integrating these diapycnal velocities into the far field yields the contribution of each term to the net diapycnal transport. Beginning with the diffusivity term $\omega_{\kappa}$,

$$
\begin{aligned}
\mathcal{E}_{\kappa}^{\infty} & =\iint_{0}^{\infty} \omega_{\kappa} \frac{|\nabla b|}{b_{y}} d z d x \\
& =\iint_{0}^{\infty} \frac{\kappa_{z}}{b_{y}} d z d x \\
& =\cot \theta \iint_{0}^{\infty} \kappa_{z}[1-r(z)] d z d x \\
& =-\cot \theta \frac{\kappa_{0}-\kappa_{\infty}}{1+\left(q_{0} \hat{d}\right)^{-1}+\frac{1}{2}\left(q_{0} \hat{d}\right)^{-2}} \int d x
\end{aligned}
$$

where $r(z)=e^{-q_{0} z}\left(\sin q_{0} z+\cos q_{0} z\right)$. The contribution of variations in the buoyancy gradient $\omega_{\nabla^{2} b}$ then follows

$$
\mathcal{E}_{\nabla^{2} b}^{\infty}=\mathcal{E}^{\infty}-\mathcal{E}_{\kappa}^{\infty}=\cot \theta \frac{\kappa_{0}+\kappa_{\infty}\left[\left(q_{0} \hat{d}\right)^{-1}+\frac{1}{2}\left(q_{0} \hat{d}\right)^{-2}\right]}{1+\left(q_{0} \hat{d}\right)^{-1}+\frac{1}{2}\left(q_{0} \hat{d}\right)^{-2}} \int d x .
$$

In the limit $q_{0}^{-1} \ll \hat{d}$ these reduce to Eqs. (24) and (25). For the isopycnal curvature term,

$$
\begin{aligned}
\mathcal{E}_{g}^{\infty} & =\iint_{0}^{\infty} \omega_{g} \frac{|\nabla b|}{b_{y}} d z d x, \\
& =b_{y} \iint_{0}^{\infty} \kappa \frac{b_{z z}}{b_{z}^{2}+b_{y}^{2}} d z d x, \\
& =-\iint_{0}^{\infty} \frac{\kappa r^{\prime} \cot \theta}{\cot ^{2} \theta(1-f)^{2}+1} d z d x .
\end{aligned}
$$

With variable $\kappa$ this integral cannot be performed analytically. However, $\omega_{g}$ is only significant in the bottom of the BBL where $\kappa \approx \kappa_{0}$ (assuming $q_{0}^{-1} \ll \hat{d}$ also ensures this). With this approximation, noting that $d / d z[\arctan (z)]=\left(1+z^{2}\right)^{-1}$, Eq. (C12) reduces to Eq. (26).

\section{REFERENCES}

Allen, J. S., and P. A. Newberger, 1998: On symmetric instabilities in oceanic bottom boundary layers. J. Phys. Oceanogr., 28, 1131-1151, https://doi.org/10.1175/1520-0485(1998)028<1131: OSIIOB $>2.0 . \mathrm{CO} ; 2$.

Burns, K. J., G. M. Vasil, J. S. Oishi, D. Lecoanet, and B. P. Brown, 2020: Dedalus: A flexible framework for numerical simulations with spectral methods. Phys. Rev. Res., 2, 023068, https:// doi.org/10.1103/PhysRevResearch.2.023068.

Callies, J., 2018: Restratification of abyssal mixing layers by submesoscale baroclinic eddies. J. Phys. Oceanogr., 48, 19952010, https://doi.org/10.1175/JPO-D-18-0082.1.

de Lavergne, C., G. Madec, J. Le Sommer, A. J. G. Nurser, and A. C. Naveira Garabato, 2016: On the consumption of Antarctic bottom water in the Abyssal ocean. J. Phys. Oceanogr., 46, 635661, https://doi.org/10.1175/JPO-D-14-0201.1.

- — - F. Roquet, R. M. Holmes, and T. J. McDougall, 2017: Abyssal ocean overturning shaped by seafloor distribution. Nature, 551, 181-186, https://doi.org/10.1038/nature24472.
Dell, R. W., and L. J. Pratt, 2015: Diffusive boundary layers over varying topography. J. Fluid Mech., 769, 635-653, https:// doi.org/10.1017/jfm.2015.88.

Ferrari, R., A. Mashayek, T. J. McDougall, M. Nikurashin, and J.-M. Campin, 2016: Turning ocean mixing upside down. J. Phys. Oceanogr., 46, 2239-2261, https://doi.org/10.1175/ JPO-D-15-0244.1.

Garrett, C., 1990: The role of secondary circulation in boundary mixing. J. Geophys. Res., 95, 3181-3188, https://doi.org/10.1029/ JC095iC03p03181.

_- 1991: Marginal mixing theories. Atmos.-Ocean, 29, 313-339, https://doi.org/10.1080/07055900.1991.9649407.

_ 2001: An isopycnal view of near-boundary mixing and associated flows. J. Phys. Oceanogr., 31, 138-142, https://doi.org/ 10.1175/1520-0485(2001)031<0138:AIVONB > 2.0.CO;2.

— P. MacCready, and P. Rhines, 1993: Boundary mixing and arrested Ekman layers: Rotating stratified flow near a sloping boundary. Annu. Rev. Fluid Mech., 25, 291-323, https:// doi.org/10.1146/annurev.fl.25.010193.001451.

Greatbatch, R. J., and K. G. Lamb, 1990: On parameterizing vertical mixing of momentum in non-eddy resolving ocean models. J. Phys. Oceanogr., 20, 1634-1637, https://doi.org/ 10.1175/1520-0485(1990)020<1634:OPVMOM >2.0.CO;2.

Holmes, R. M., C. de Lavergne, and T. J. McDougall, 2018: Ridges, seamounts, troughs, and bowls: Topographic control of the dianeutral circulation in the abyssal ocean. J. Phys. Oceanogr., 48, 861-882, https://doi.org/10.1175/JPO-D-17-0141.1.

,-- , and -2019 : Tracer transport within abyssal mixing layers. J. Phys. Oceanogr., 49, 2669-2695, https://doi.org/ 10.1175/JPO-D-19-0006.1.

Holtappels, M., and A. Lorke, 2011: Estimating turbulent diffusion in a benthic boundary layer. Limnol. Oceanogr. Methods, $\mathbf{9}$, 29-41, https://doi.org/10.4319/lom.2011.9.29.

Kunze, E., C. MacKay, E. E. McPhee-Shaw, K. Morrice, J. B. Girton, and S. R. Terker, 2012: Turbulent mixing and exchange with interior waters on sloping boundaries. J. Phys. Oceanogr., 42, 910-927, https://doi.org/10.1175/JPO-D-11-075.1.

Marshall, J., D. Jamous, and J. Nilsson, 1999: Reconciling thermodynamic and dynamic methods of computation of watermass transformation rates. Deep-Sea Res. I, 46, 545-572, https://doi.org/10.1016/S0967-0637(98)00082-X.

McDougall, T. J., and R. Ferrari, 2017: Abyssal upwelling and downwelling driven by near-boundary mixing. J. Phys. Oceanogr., 47, 261-283, https://doi.org/10.1175/JPO-D-16-0082.1.

McPhee-Shaw, E., 2006: Boundary-interior exchange: Reviewing the idea that internal-wave mixing enhances lateral dispersal near continental margins. Deep-Sea Res. II, 53, 42-59, https:// doi.org/10.1016/j.dsr2.2005.10.018.

Phillips, O., J.-H. Shyu, and H. Salmun, 1986: An experiment on boundary mixing: Mean circulation and transport rates. J. Fluid Mech., 173, 473-499, https://doi.org/10.1017/S0022112086001234.

Polzin, K. L., J. M. Toole, J. R. Ledwell, and R. W. Schmitt, 1997: Spatial variability of turbulent mixing in the abyssal ocean. Science, 276, 93-96, https://doi.org/10.1126/science.276.5309.93.

Rhines, P. B., and W. R. Young, 1982: Homogenization of potential vorticity in planetary gyres. J. Fluid Mech., 122, 347-367, https://doi.org/10.1017/S0022112082002250.

Ruan, X., and J. Callies, 2020: Mixing-driven mean flows and submesoscale eddies over mid-ocean ridge flanks and fracture zone canyons. J. Phys. Oceanogr., 50, 175-195, https://doi.org/ 10.1175/JPO-D-19-0174.1.

_- A. F. Thompson, and J. R. Taylor, 2019: The evolution and arrest of a turbulent stratified oceanic bottom boundary 
layer over a slope: Downslope regime. J. Phys. Oceanogr., 49, 469-487, https://doi.org/10.1175/JPO-D-18-0079.1.

Thorpe, S. A., 1987: Current and temperature variability on the continental slope. Philos. Trans. Roy. Soc. London, A323, 471-517, https://doi.org/10.1098/rsta.1987.0100.

Thurnherr, A., L. Clément, L. S. Laurent, R. Ferrari, and T. Ijichi, 2020: Transformation and upwelling of bottom water in fracture zone valleys. J. Phys. Oceanogr., 50, 715-726, https:// doi.org/10.1175/JPO-D-19-0021.1.

Toole, J. M., R. W. Schmitt, and K. L. Polzin, 1994: Estimates of diapycnal mixing in the abyssal ocean. Science, 264, 11201123, https://doi.org/10.1126/science.264.5162.1120.

Umlauf, L., and H. Burchard, 2011: Diapycnal transport and mixing efficiency in stratified boundary layers near sloping topography. J. Phys. Oceanogr., 41, 329-345, https://doi.org/ 10.1175/2010JPO4438.1.

, W. D. Smyth, and J. N. Moum, 2015: Energetics of bottom Ekman layers during buoyancy arrest. J. Phys. Oceanogr., 45, 3099-3117, https://doi.org/10.1175/JPO-D-15-0041.1. van Haren, H., 2017: Exploring the vertical extent of breaking internal wave turbulence above deep-sea topography. Dyn. Atmos. Oceans, 77, 89-99, https://doi.org/10.1016/j.dynatmoce.2017.01.002.

Waterhouse, A. F., and Coauthors, 2014: Global patterns of diapycnal mixing from measurements of the turbulent dissipation rate. J. Phys. Oceanogr., 44, 1854-1872, https://doi.org/10.1175/ JPO-D-13-0104.1.

Wenegrat, J. O., J. Callies, and L. N. Thomas, 2018: Submesoscale baroclinic instability in the bottom boundary layer. J. Phys. Oceanogr., 48, 2571-2592, https://doi.org/10.1175/JPO-D-17-0264.1.

Winters, K. B., 2015: Tidally driven mixing and dissipation in the stratified boundary layer above steep submarine topography. Geophys. Res. Lett., 42, 7123-7130, https://doi.org/10.1002/2015GL064676.

Wüest, A., and A. Lorke, 2003: Small-scale hydrodynamics in lakes. Annu. Rev. Fluid Mech., 35, 373-412, https://doi.org/ 10.1146/annurev.fluid.35.101101.161220.

Wunsch, C., 1970: On oceanic boundary mixing. Deep-Sea Res. Oceanogr. Abstr., 17, 293-301, https://doi.org/10.1016/00117471(70)90022-7. 